\title{
Effects of voriconazole on population pharmacokinetics and optimization of the initial dose of tacrolimus in children with chronic granulomatous disease undergoing hematopoietic stem cell transplantation
}

\author{
Xiao Chen ${ }^{1 \#}$, Dongdong Wang ${ }^{1 \#}$, Jianger Lan ${ }^{1 *}$, Guangfei Wang ${ }^{1}$, Lin Zhu ${ }^{1}$, Xiaoyong Xu ${ }^{1}$, Xiaowen Zhai ${ }^{2}$, \\ Hong $\mathrm{Xu}^{3}$, Zhiping $\mathrm{Li}^{1}$ \\ ${ }^{1}$ Department of Pharmacy, Children's Hospital of Fudan University, National Children's Medical Center, Shanghai, China; ${ }^{2}$ Department of \\ Hematology and Oncology, Children's Hospital of Fudan University, National Children's Medical Center, Shanghai, China; ${ }^{3}$ Department of \\ Nephrology, Children's Hospital of Fudan University, National Children's Medical Center, Shanghai, China \\ Contributions: (I) Conception and design: X Chen, D Wang, X Zhai, H Xu, Z Li; (II) Administrative support: X Zhai, H Xu, Z Li; (III) Provision of \\ study materials or patients: X Chen, D Wang, J Lan; (IV) Collection and assembly of data: X Chen, D Wang, J Lan, G Wang, L Zhu, X Xu; (V) Data \\ analysis and interpretation: X Chen, D Wang; (VI) Manuscript writing: All authors; (VII) Final approval of manuscript: All authors. \\ \#These authors contributed equally to this work and are co-first authors. \\ Correspondence to: Xiaowen Zhai. Department of Hematology and Oncology, Children's Hospital of Fudan University, National Children's Medical \\ Center, Shanghai 201102, China. Email: zhaixiaowendy@163.com; Hong Xu. Department of Nephrology, Children’s Hospital of Fudan University, \\ National Children’s Medical Center, Shanghai 201102, China. Email: hxu@shmu.edu.cn; Zhiping Li. Department of Pharmacy, Children's Hospital \\ of Fudan University, National Children's Medical Center, Shanghai 201102, China. Email: zpli@fudan.edu.cn.
}

Background: This study aimed to explore the effects of voriconazole on population pharmacokinetics and optimization of the initial dose of tacrolimus in children with chronic granulomatous disease (CGD) undergoing hematopoietic stem cell transplantation (HSCT).

Methods: Thirty-four children with CGD undergoing HSCT were assessed to establish a population pharmacokinetic model (PPM) using the non-linear mixed effect. Tacrolimus concentrations were simulated by the Monte Carlo method in children weighing $<25 \mathrm{~kg}$ at different doses.

Results: In the final model, weight and concomitant use of voriconazole were included as covariates. With the same weight, the relative value of tacrolimus clearance was 1:0.388 in children not taking voriconazole: children taking voriconazole. Compared with children not taking voriconazole, the measured tacrolimus concentrations were all higher in children taking voriconazole $(\mathrm{P}<0.01)$; however, these were not corrected by dose or body weight for concentration differences. Thus, we simulated the tacrolimus concentrations using different body weights $(5-25 \mathrm{~kg})$ and different dose regimens $(0.1-0.8 \mathrm{mg} / \mathrm{kg} /$ day $)$ for the same body weight and dose. Tacrolimus concentrations in children taking voriconazole were higher than those in children not taking voriconazole $(\mathrm{P}<0.01)$. Also, in children with CGD undergoing HSCT who were not taking voriconazole, the initial dose regimen of $0.5 \mathrm{mg} / \mathrm{kg} /$ day was recommended for body weights of $5-10 \mathrm{~kg}$, and $0.4 \mathrm{mg} / \mathrm{kg} / \mathrm{day}$ was recommended for body weights of 10-25 kg. In children with CGD undergoing HSCT who were taking voriconazole, an initial dose regimen of $0.3 \mathrm{mg} / \mathrm{kg} /$ day was recommended for body weights of $5-25 \mathrm{~kg}$.

Conclusions: We established, for the first time, a PPM of tacrolimus in children with CGD undergoing HSCT in which voriconazole significantly increased tacrolimus concentrations. In addition, the initial dose of tacrolimus in children with CGD undergoing HSCT was recommended.

Keywords: Voriconazole; tacrolimus; chronic granulomatous disease; hematopoietic stem cell transplantation

Submitted Jul 28, 2021. Accepted for publication Sep 10, 2021.

doi: 10.21037/atm-21-4124

View this article at: https://dx.doi.org/10.21037/atm-21-4124 


\section{Introduction}

Chronic granulomatous disease (CGD), a rare primary immunodeficiency, affects 1 in 200,000-250,000 individuals (1). It is characterized by susceptibility to typical pathogens and is accompanied by recurrent infections as well as inflammatory and autoimmune complications (2-4). The diagnosis median age is 2.7-3.0 years (4-7), which can result in severe and life-threatening infections in affected children (8).

CGD involves a defect in the phagocytic system. The nicotinamide adenine dinucleotide phosphate (NADPH) oxidase complex generates reactive oxygen species (ROS), which are essential for killing pathogenic microorganisms (especially catalase-positive bacteria and fungi) (9). In CGD, the NADPH oxidase complex is dysfunctional, and CGD patients are more likely to suffer from autoimmune disorders, such as liver-function abnormalities, hollowviscera obstruction, and inflammatory bowel disease, which exhibit multiple-system involvement $(2,10,11)$.

Hematopoietic stem cell transplantation (HSCT) is the first-line treatment for CGD (12-14). HSCT may adjust superoxide generation, making neutrophil function within normal range and improvement in inflammatory progression (14). However, HSCT patients must take tacrolimus, a potent immunosuppressant, for a prolonged period to prevent transplant rejection (15-18). In addition, voriconazole, a second-generation triazole with potent broad-spectrum antifungal activity, is used for prophylaxis against invasive fungal disease (IFD), which is a major cause of morbidity and mortality in pediatric patients after HSCT $(19,20)$. It has been reported that the concomitant use of voriconazole has effects on tacrolimus $(21,22)$. However, the effects of voriconazole on population pharmacokinetics and optimization of the initial dose of tacrolimus in patients with CGD patients undergoing HSCT (especially children) remain unknown. Population pharmacokinetics mainly describes the dispersion degree and distribution of pharmacokinetic parameters from the patient population, explores the influencing factors of pharmacokinetics, and provides strong help for individual drug administration of patients.

In this study, we explored the effects of voriconazole on population pharmacokinetics and optimization of the initial dose of tacrolimus in children with CGD undergoing HSCT. We present the following article in accordance with the MDAR reporting checklist (available at https://dx.doi. org/10.21037/atm-21-4124).

\section{Methods}

\section{Ethical approval of the study protocol}

This was a retrospective study. All procedures performed in this study involving human participants were in accordance with the Declaration of Helsinki (as revised in 2013). The study protocol was approved by the ethics committee of the Children's Hospital of Fudan University (Shanghai, China) ([2019] 020). Individual consent for this retrospective analysis was waived.

\section{Data collection}

The data of children with CGD undergoing HSCT treated with tacrolimus from May 2016 to January 2021 at the Children's Hospital of Fudan University were retrospectively collected.

Tacrolimus concentrations were tested using the Emit ${ }^{\circledR}$ 2000 Tacrolimus Assay (Siemens Healthcare Diagnostics, Newark, NJ, USA) with a range of 2.0-30 $\mathrm{ng} / \mathrm{mL}$. In addition, we collected the demographic data (sex, age, body weight), clinical and biochemistry results (albumin, alanine transaminase, aspartate transaminase, creatinine, urea, total protein, total bile acid, direct bilirubin, total bilirubin, hematocrit, hemoglobin, mean corpuscular hemoglobin, and mean corpuscular hemoglobin concentration), and concurrent use of other medications (caspofungin, ethambutol, glucocorticoids, isoniazid, micafungin, mycophenolic acid, omeprazole, vancomycin, and voriconazole). A partial basic clinical dataset of some children were collected from a previous study (23).

\section{Population pharmacokinetic model (PPM)}

PPM mainly describes the dispersion degree and distribution of pharmacokinetic parameters from the patient population, explores the influencing factors of pharmacokinetics, and provides strong help for individual drug administration of patients. The non-linear, mixed-effects modeling software NONMEM v7 (Icon Development Solutions, Ellicott City, MD, USA) and a first-order conditional estimation method with interaction (FOCE-I) approach were used to establish the PPM of tacrolimus in children with CGD undergoing HSCT. The pharmacokinetic parameters included the apparent oral clearance $(\mathrm{CL} / \mathrm{F})$, volume of distribution $(\mathrm{V} / \mathrm{F})$, and absorption rate constant $\left(\mathrm{K}_{\mathrm{a}}\right)$, which was fixed at 4.48/h (24,25). 


\section{Random-effect model}

The inter-individual variability was estimated using Eq. [1]

$$
S_{i}=T V(S) \times \exp \left(\eta_{i}\right)
$$

where $S_{i}$ is the individual parameter value, $\operatorname{TV}(S)$ is a typical individual parameter value, and $\eta_{\mathrm{i}}$ is the symmetrical distribution, which was a random term with a zero mean and variance of $\omega^{2}$.

The random residual variability was estimated using Eq. [2]:

$$
Q_{i}=I P C_{i} \times\left(1+\varepsilon_{1}\right)+\varepsilon_{2}
$$

where $\mathrm{O}_{\mathrm{i}}$ is the observed concentration, $\mathrm{IPC}_{\mathrm{i}}$ is the individual predicted concentration, and $\varepsilon_{1}$ and $\varepsilon_{2}$ are the symmetrical distribution, which were random terms with a zero mean and variance of $\sigma^{2}$.

\section{Covariate model}

The pharmacokinetic parameters and body weight were estimated using Eq. [3]

$$
C_{i}=C_{\text {std }} \times\left(X_{i} / X_{\text {std }}\right)^{\text {power }}
$$

where $\mathrm{C}_{\mathrm{i}}$ is the $\mathrm{i}$-th individual parameter, $\mathrm{C}_{\text {std }}$ is a typical parameter, $X_{i}$ is the $i$-th individual body weight, $X_{\text {std }}$ is the standard body weight of $70 \mathrm{~kg}$, and power was the allometric coefficient: 0.75 for CL/F and 1 for V/F (26).

The pharmacokinetic parameters and continuous covariates or categorical covariates were estimated using Eqs. [4] and [5]:

$$
\begin{aligned}
& S_{i}=T V(S) \times\left(\operatorname{Cov}_{i} / \text { Covmedian }^{\theta}\right. \\
& S_{i}=T V(S) \times\left(1+\theta \times \operatorname{Cov}_{i}\right)
\end{aligned}
$$

where $S_{i}$ is the individual parameter value, $\operatorname{TV}(S)$ is a typical individual parameter value, $\theta$ is the parameter to be estimated, $\mathrm{Cov}_{\mathrm{i}}$ is the covariate of the $\mathrm{i}$-th individual, and $\mathrm{Cov}_{\text {median }}$ is the population median for the covariate.

\section{Statistical analysis}

Objective function value (OFV) changes were used as the inclusion criteria for covariates; a decrease in the OFV $>3.84(\mathrm{P}<0.05)$ was the inclusion standard, while an increase in the $\mathrm{OFV}>6.63(\mathrm{P}<0.01)$ was the exclusion standard.

\section{Model evaluation}

The model was evaluated using the following: observations $v s$. population predictions, observations $v s$. individual predictions, conditional weighted residuals (WRES) $v s$. population predictions, conditional WRES $v$ s. time after the start of therapy, a visual predictive check (VPC) of the model, and individual plots. In addition, model stability was evaluated using 1,000 bootstraps with different random sampling.

\section{Simulation}

Two scenarios were simulated to acquire tacrolimus concentrations: concomitant use of voriconazole or no concomitant use of voriconazole. In each scenario, 1,000 "virtual" children with CGD undergoing HSCT were simulated in five body weight groups $(5,10,15,20$, and $25 \mathrm{~kg})$ for eight doses $(0.1,0.2,0.3,0.4,0.5,0.6,0.7$, and $0.8 \mathrm{mg} / \mathrm{kg} /$ day), which were divided evenly into two doses. In addition, the effects of tacrolimus initial dosage on target concentration $(5-20 \mathrm{ng} / \mathrm{mL})$ were explored by Monte Carlo simulations on the basis of final model.

\section{Results}

\section{Patient information}

Thirty-four children (age range, $2.29 \pm 1.89$ years) with CGD undergoing HSCT were included in the analyses. The demographic data of patients and drug combinations are shown in Table 1. A partial basic clinical dataset of some children were collected from a previous study (23). A total of 293 tacrolimus concentrations were included in the present study, and the mean number of concentrations per patient was 8.6.

\section{Modeling and evaluation}

The final models were:

$$
\begin{aligned}
& C L / F=35.4 \times(W T / 70)^{0.75} \times(1-0.612 \times V R C) \\
& V / F=5970 \times(W T / 70)
\end{aligned}
$$

where WT denotes body weight, and VRC refers to voriconazole. If a patient took voriconazole, then the VRC value was 1 ; otherwise the VRC value was 0 .

Model evaluation is shown in Figure 1. Figure 1A-1E display the observations $v s$. population predictions, observations $v s$. individual predictions, conditional WRES $v s$. population predictions, conditional WRES $v s$. time after 
Table 1 Demographic data of patients and drug combination ( $\mathrm{n}=34)$

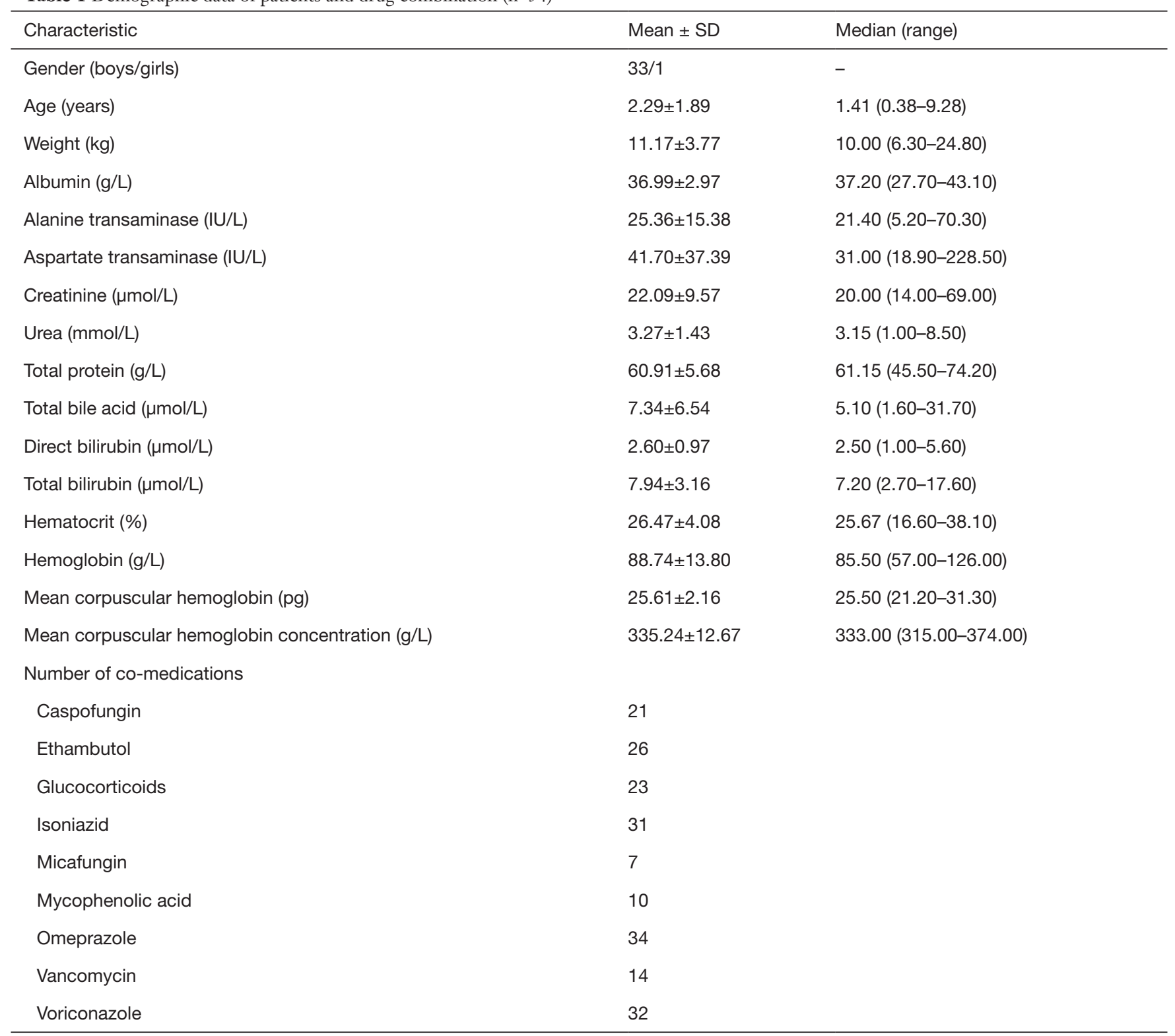

the start of therapy, and VPC of the model, respectively. The final model had good performance according to Figure $1 A-1 D$. VPC revealed that most of the observed concentrations were within the $95 \%$ prediction intervals of the simulation data. Hence, the prediction-corrected concentrations were well served by the final model. Figure 2 shows the individual plots, and demonstrated that the final model had acceptable predictability.

The final model's parameter estimates and bootstrap validation are shown in Table 2 . The median values of the 1,000 bootstraps were close to the final model's respective parameter values, showing that the model was accurate and reliable.

\section{Effects of voriconazole on tacrolimus in children with CGD undergoing HSCT}

Tacrolimus CL/F in children with CGD undergoing HSCT is shown in Figure 3. Under the equal weight, tacrolimus $\mathrm{CL} / \mathrm{F}$ was 1:0.388 in children not taking voriconazole: children taking voriconazole. Compared with children not taking voriconazole, the measured tacrolimus concentrations 

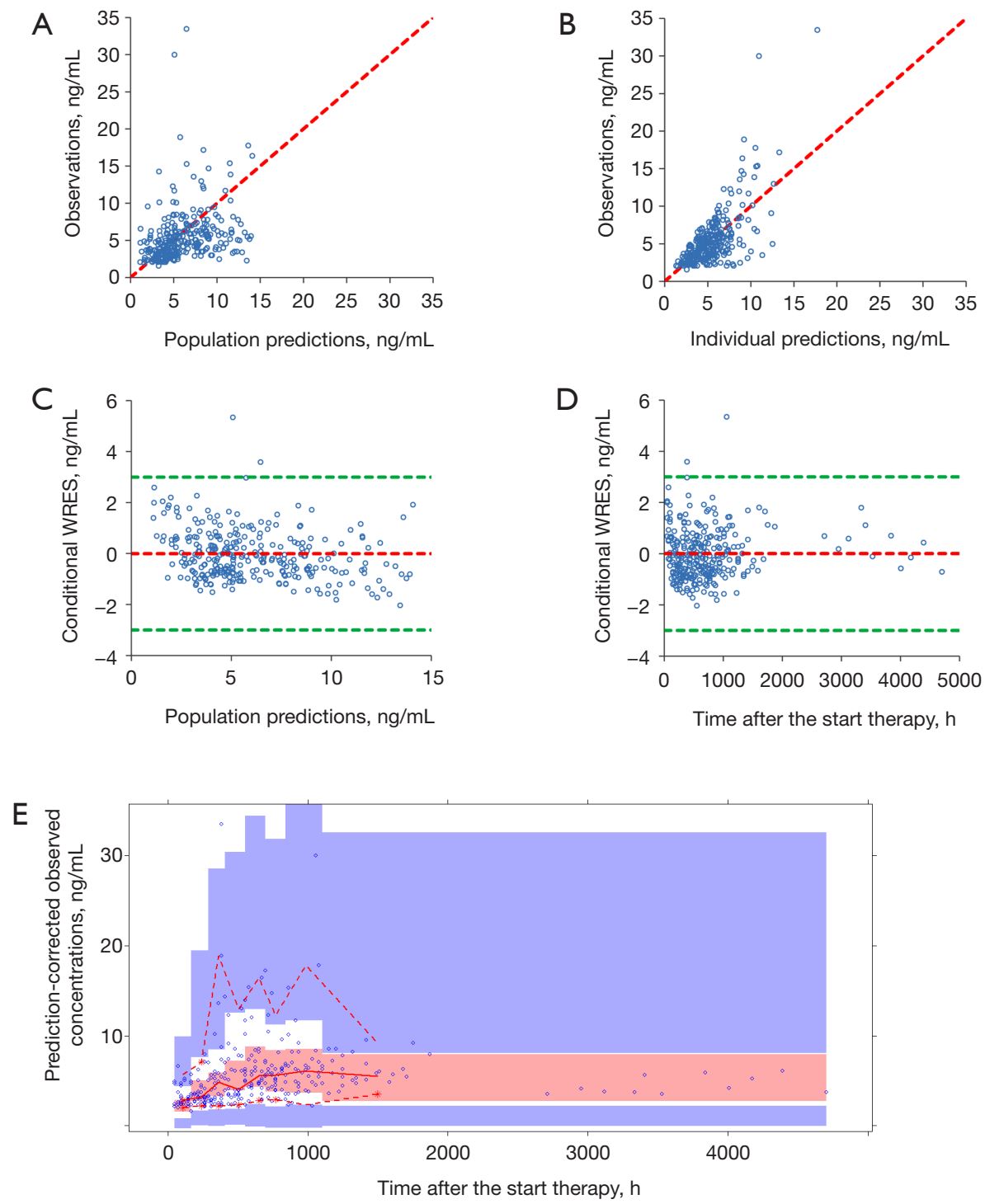

Figure 1 Model evaluation. (A) Observations vs. population predictions; (B) observations vs. individual predictions; (C) conditional WRES $v s$. population predictions; (D) conditional WRES $v s$. time after the start of therapy; (E) VPC of model. The middle solid line represents the median of the prediction-corrected concentrations. The lower and upper dashed lines are the 2.5 th and 97.5 th percentiles of the predictioncorrected concentrations. Partial concentration values were collected in previous study (23). WRES, weighted residuals; VPC, visual predictive check.

were all higher in children taking voriconazole $(\mathrm{P}<0.01)$; however, these were not corrected by dose or body weight for concentration differences.

Thus, we further simulated the tacrolimus concentrations for different body weights $(5-25 \mathrm{~kg})$ and dose regimens $(0.1-0.8 \mathrm{mg} / \mathrm{kg} /$ day) (Figure 4). For an identical body weight and dose, tacrolimus concentrations in children taking voriconazole were higher than those in children not taking voriconazole $(\mathrm{P}<0.01)$. These results suggested that voriconazole significantly increased the tacrolimus concentrations in children with CGD undergoing HSCT. Hence, attention should be paid to adjustment of the drug dose to prevent toxicity if these two drugs are combined.

\section{Optimization of the initial dose of tacrolimus in children with CGD undergoing HSCT}

Figure 5 shows the probability of achieving the target 

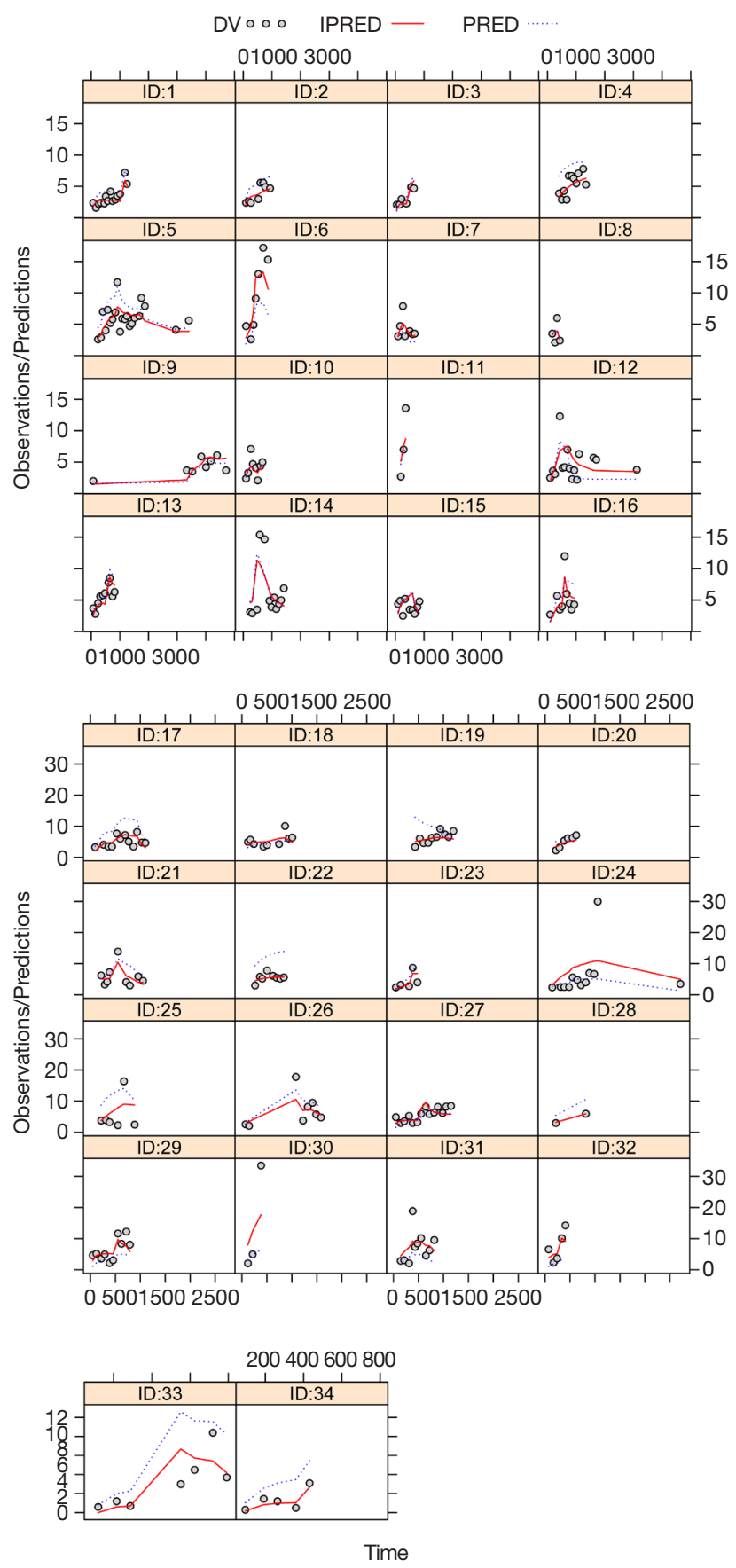

Figure 2 Individual plots. ID, patient ID number; DV, measured concentration value; IPRED, individual predictive value; PRED, population predictive value.

concentrations with different tacrolimus initial dosages. Figure $5 \mathrm{~A}$ shows the data for children with CGD undergoing HSCT who were not taking voriconazole. Figure $5 B$ shows the results for children with CGD undergoing HSCT who were administered voriconazole. In children with CGD undergoing HSCT who were not taking voriconazole, an initial dose of $0.5 \mathrm{mg} / \mathrm{kg} / \mathrm{day}$ was recommended for body weights of $5-10 \mathrm{~kg}$, and $0.4 \mathrm{mg} / \mathrm{kg} / \mathrm{day}$ was recommended for body weights of 10-25 kg. In children with CGD undergoing HSCT who were taking voriconazole, an initial dose of $0.3 \mathrm{mg} / \mathrm{kg} /$ day was recommended for body weights of $5-25 \mathrm{~kg}$.

\section{Discussion}

Conventional treatment for CGD is antifungal and antibacterial prophylaxis with azoles and cotrimoxazole, and immunosuppressive therapy $(27,28)$. Prophylaxis leads to a significant reduction in death rates, but infections reportedly occur at a rate of $0.26-0.64$ per patient-year $(29,30)$, whose cumulative lifetime risk for aspergillosis of $20-40 \%$ being the leading cause of death (27). Martire et al. reported the median lifespan of CGD patients receiving conventional treatment to be 30-40 years (29), which was mainly relied on the NADPH oxidase's residual activity (5). Other treatment options, such as long-term use of corticosteroids (which are immunosuppressants), further increase the risk of infections and failure to thrive $(27,28)$.

Fortunately, HSCT can cure CGD with resolution of infections and inflammatory complications (31-35). Additionally, compared with those treated conservatively, the growth and quality of life of transplanted patients are improved (30,36). For example, Arnold et al. reported on HSCT in adolescent patients with CGD (37). Tang et al. demonstrated that HSCT using unrelated cord blood or unmanipulated haploidentical donors was efficacious against pediatric CGD with inflammatory complications and severe infection (38). Rocha et al. showed successful immune reconstitution by means of HSCT in a Colombian patient with CGD (39).

Nevertheless, HSCT has limitations. High-risk patients with ongoing infectious or active inflammatory complications at HSCT initiation have considerable transplant-related mortality $(\leq 38 \%)(31,32)$. In addition, Dedieu et al. reported that CGD patients undergoing HSCT until 8 years of age showed excellent survival, but young children needed more intense conditioning to avoid graft rejection (27). Therefore, reducing infection while resisting graft-versus-host disease (GVHD) is particularly important $(31,40,41)$.

Tacrolimus is a first-line agent for GVHD prevention 
Table 2 Parameter estimates of final model and bootstrap validation

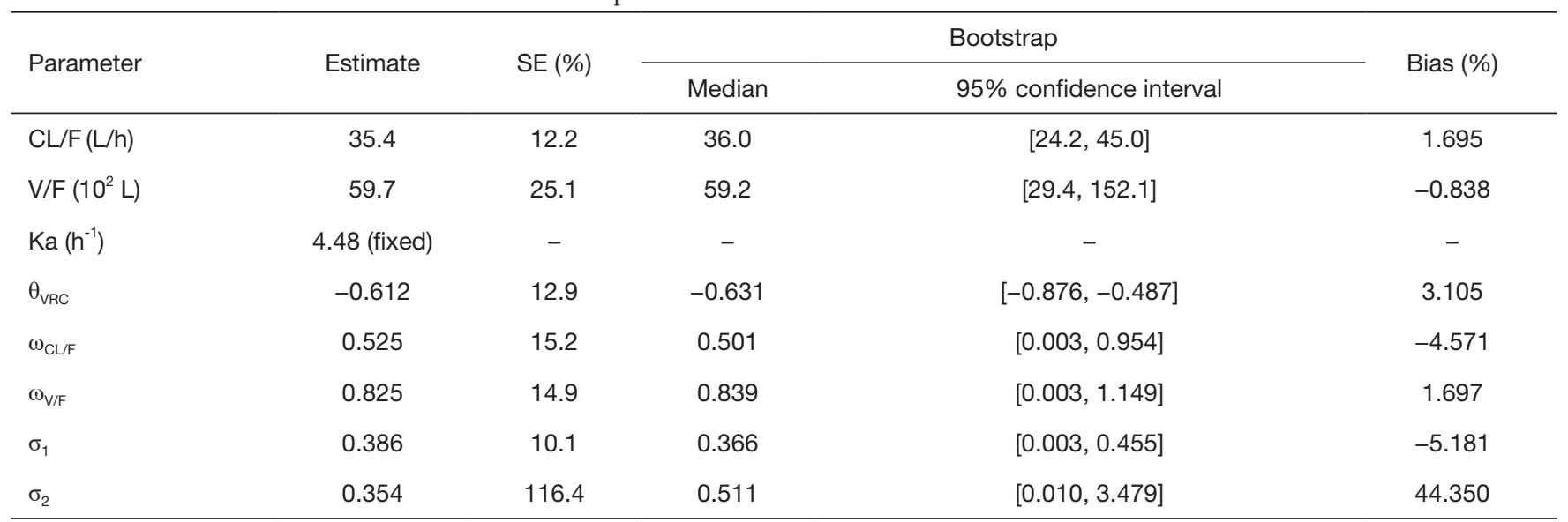

$95 \%$ confidential interval was displayed as the $2.5^{\text {th }}$ and 97.5 th percentiles of bootstrap estimates. CL/F, apparent oral clearance (L/h); V/ $\mathrm{F}$, apparent volume of distribution (L); Ka, absorption rate constant $\left(\mathrm{h}^{-1}\right) ; \theta_{\mathrm{VRC}}$ was the coefficient of the voriconazole; $\omega_{\mathrm{CLF}}$, inter-individual variability of $\mathrm{CL} / \mathrm{F} ; \omega_{\mathrm{V} / \mathrm{F}}$, inter-individual variability of V/F; $\sigma_{1}$, residual variability, proportional error; $\sigma_{2}$, residual variability, additive error; Bias, prediction error, Bias $=($ Median - Estimate $) /$ Estimate $\times 100 \%$.

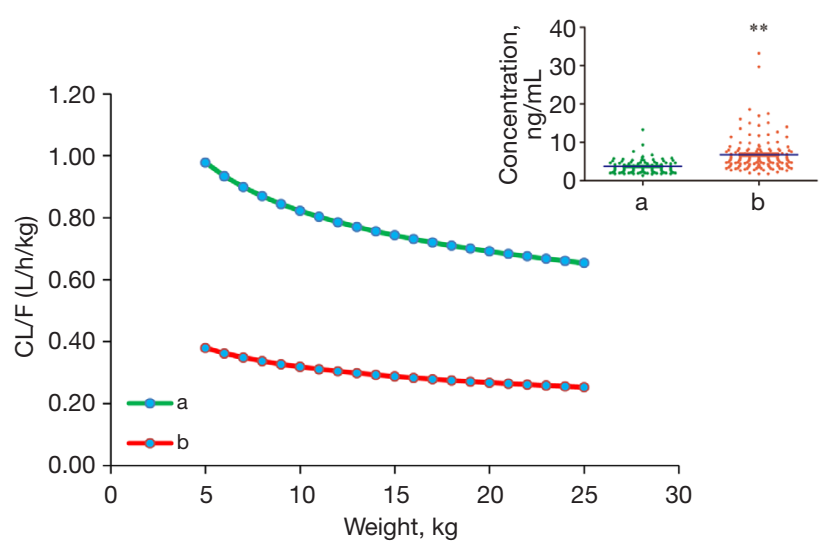

Figure 3 Tacrolimus CL/F in children with CGD undergoing HSCT. a: without voriconazole; b: with voriconazole. ${ }^{* *}$, $\mathrm{P}<0.01$ vs. children without voriconazole (measured tacrolimus concentrations). CGD, chronic granulomatous disease; HSCT, hematopoietic stem cell transplantation.

and has been routinely employed to stop rejection of HSCT (15-18). However, tacrolimus has a narrow therapeutic range; the use of high concentrations seems to be related to toxicity, while lower concentrations are related to an acute rejection's increased risk (23). More importantly, some combinations of clinical drugs have been shown to affect the population pharmacokinetic process of tacrolimus in vivo $(42,43)$, thereby influencing its efficacy or producing toxicity. Therefore, it is particularly important to study the population pharmacokinetic process of tacrolimus in children with CGD as well as its influencing factors.

In the present study, 34 patients were enrolled, which were enough for population pharmacokinetics. For example, in Yonwises et al.'s study, population pharmacokinetics of meropenem in critically ill infant patients, 35 patients were enrolled (44). In Nassar-Sheikh Rashid et al.'s study, population pharmacokinetics of infliximab in children with juvenile idiopathic arthritis, 27 patients were enrolled (45). In Hao et al.'s study, population pharmacokinetics of tacrolimus in children with nephrotic syndrome, 28 patients were enrolled (46). In Zhao et al.'s study, population pharmacokinetics and bayesian estimator of mycophenolic acid in children with idiopathic nephrotic syndrome, 23 patients were enrolled (47).

In addition, body weight and concomitant use of voriconazole were included as covariates. Voriconazole is used for prophylaxis against IFD, which is a major cause of morbidity and mortality in pediatric patients after HSCT $(19,20)$. With the same body weight, we observed that the relative value of $\mathrm{CL} / \mathrm{F}$ for tacrolimus was 1:0.388 in children not taking voriconazole: children taking voriconazole. Also, compared with children not taking voriconazole, the measured tacrolimus concentrations were all higher in children who were taking voriconazole; however, these were not corrected by dose or body weight for concentration differences. Thus, we further simulated the tacrolimus concentrations for different body weights and doses. Under an identical body weight and dose, tacrolimus concentrations in children taking voriconazole were higher 


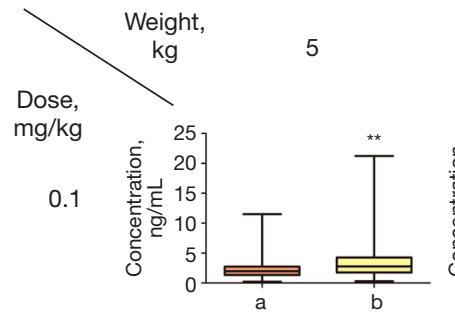

10

15

20

25
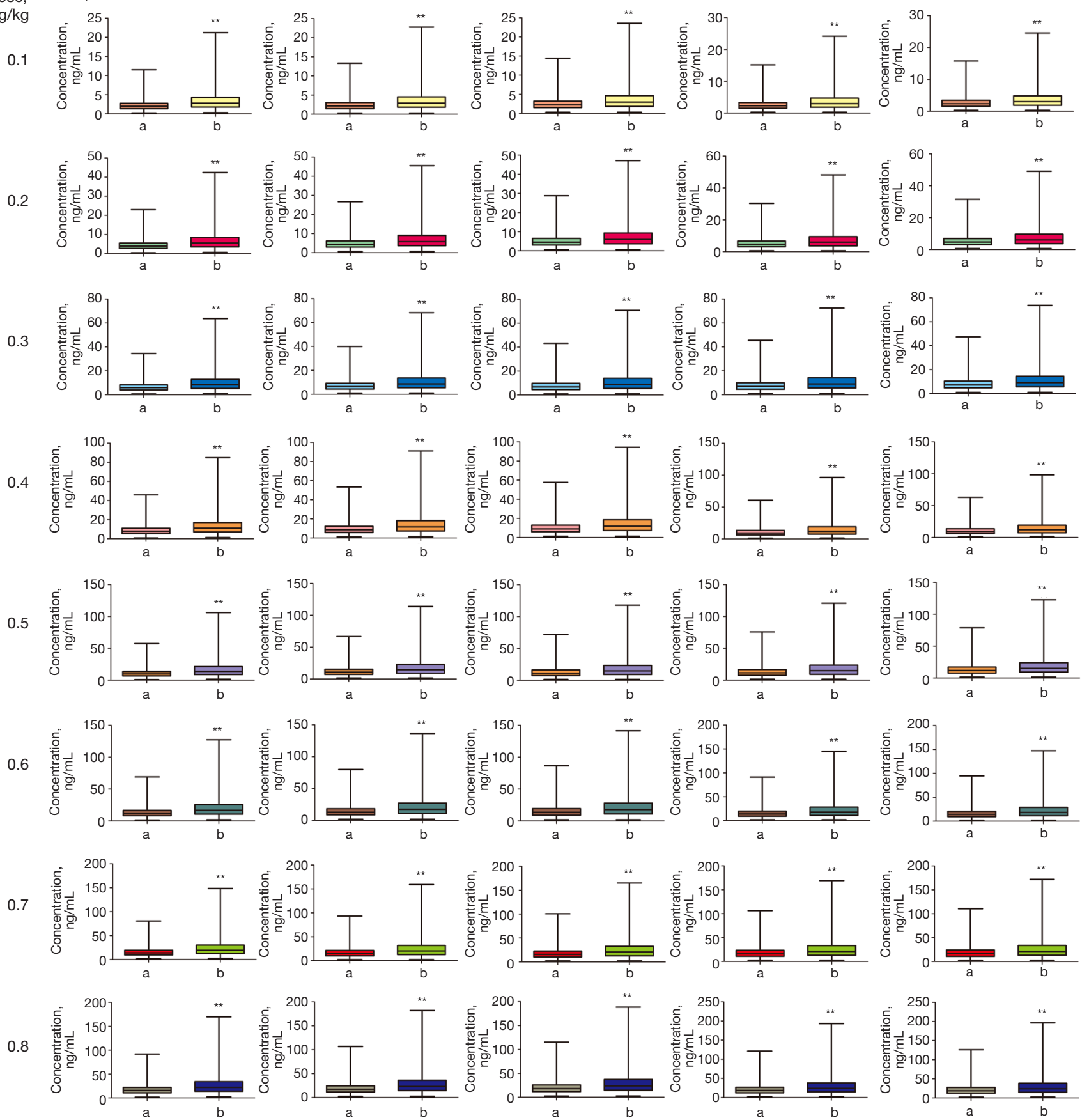

Figure 4 Effects of voriconazole on tacrolimus concentrations. a: without voriconazole; b: with voriconazole. ${ }^{* *}, \mathrm{P}<0.01$ vs. children without voriconazole. 

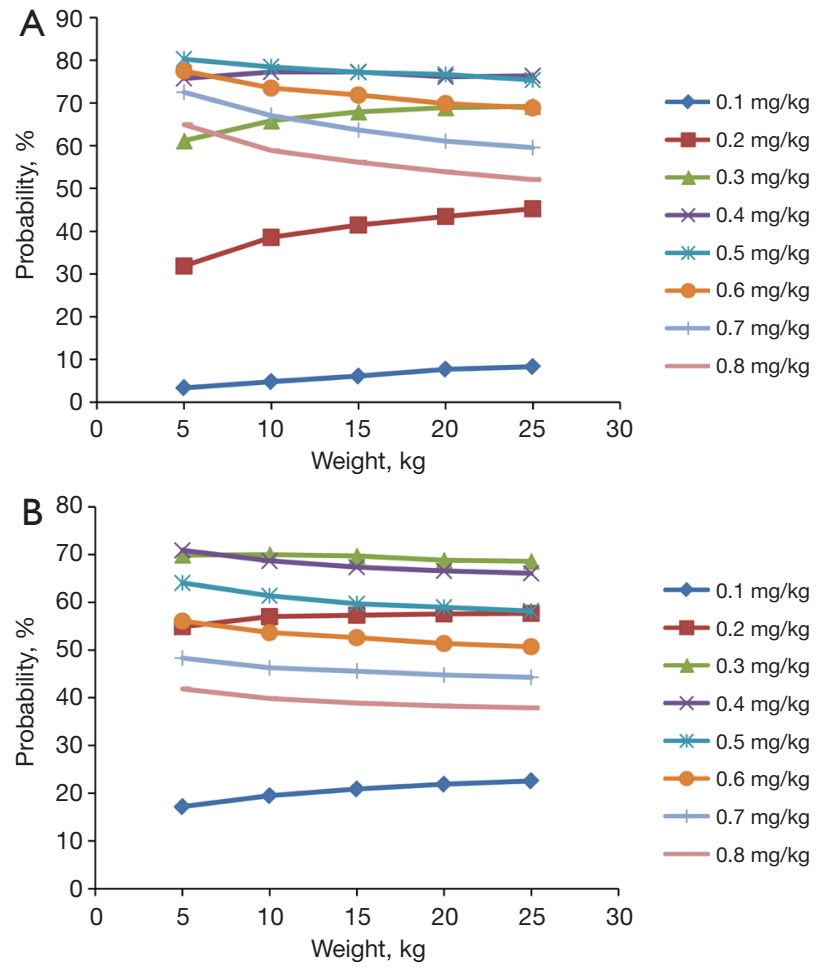

Figure 5 Probability of reaching tacrolimus concentrations. (A) CGD children undergoing HSCT without voriconazole; (B) CGD children undergoing HSCT with voriconazole. CGD, chronic granulomatous disease; HSCT, hematopoietic stem cell transplantation.

than those in children not taking voriconazole. In addition, in children with CGD undergoing HSCT who were not taking voriconazole, an initial dose of $0.5 \mathrm{mg} / \mathrm{kg} / \mathrm{day}$ was recommended for body weights of $5-10 \mathrm{~kg}$, and $0.4 \mathrm{mg} / \mathrm{kg} /$ day was recommended for children of body weight $10-25 \mathrm{~kg}$. In children with CGD undergoing HSCT who were taking voriconazole, an initial dose of $0.3 \mathrm{mg} / \mathrm{kg} /$ day was recommended for body weights of $5-25 \mathrm{~kg}$. Hence, the tacrolimus dose should be reduced if it is combined with voriconazole in children with CGD undergoing HSCT.

Our study had limitations: on the one hand, almost all of the children in the study cohort were boys. However, according to population pharmacokinetic studies of tacrolimus in pediatric patients, sex does not have a significant influence on the CL/F of tacrolimus $(46,48)$. Therefore, the sex distribution in our study cohort had little influence on our conclusions. On the other hand, this was a retrospective study and some data were not available.
For example, ROS is essential for killing pathogenic microorganisms in the treatment but without the concentration of ROS in serum in the retrospective analysis. These need be refined in future prospective studies.

\section{Conclusions}

We established, for the first time, a PPM of tacrolimus in children with CGD undergoing HSCT in which voriconazole use significantly increased tacrolimus concentrations. In addition, the initial dose of tacrolimus in children with CGD undergoing HSCT was recommended.

\section{Acknowledgments}

Funding: This work was supported by the Shanghai Municipal Education Commission (No. HJW-R-2019-66-19), the Clinical Pharmacy Key Specialty Construction Project of Shanghai (No. YZ2017/5), the Fusion Innovation Project of Xuzhou Medical University (No. XYRHCX2021011), and the Scientific research project of Science and Technology Commission of Shanghai Municipality (No. 18DZ1910604, No. 19XD1400900).

\section{Footnote}

Reporting Checklist: The authors have completed the MDAR reporting checklist. Available at https://dx.doi. org/10.21037/atm-21-4124

Data Sharing Statement: Available at https://dx.doi. org/10.21037/atm-21-4124

Conflicts of Interest: All authors have completed the ICMJE uniform disclosure form (available at https://dx.doi. org/10.21037/atm-21-4124). The authors have no conflicts of interest to declare.

Ethical Statement: The authors are accountable for all aspects of the work in ensuring that questions related to the accuracy or integrity of any part of the work are appropriately investigated and resolved. All procedures performed in this study involving human participants were in accordance with the Declaration of Helsinki (as revised in 2013). The study protocol was approved by the ethics committee of the Children's Hospital of Fudan University (Shanghai, China) ([2019] 020). Individual consent for this retrospective analysis was waived. 
Open Access Statement: This is an Open Access article distributed in accordance with the Creative Commons Attribution-NonCommercial-NoDerivs 4.0 International License (CC BY-NC-ND 4.0), which permits the noncommercial replication and distribution of the article with the strict proviso that no changes or edits are made and the original work is properly cited (including links to both the formal publication through the relevant DOI and the license). See: https://creativecommons.org/licenses/by-nc-nd/4.0/.

\section{References}

1. Bhattad S, Raghuram CP, Porta F, et al. Successful Haploidentical Transplant Using Post-Transplant Cyclophosphamide in a Child with Chronic Granulomatous Disease-First Report from the Indian Subcontinent. J Clin Immunol 2021;41:820-4.

2. Holland SM. Chronic granulomatous disease. Clin Rev Allergy Immunol 2010;38:3-10.

3. Johnston RB Jr. Clinical aspects of chronic granulomatous disease. Curr Opin Hematol 2001;8:17-22.

4. Barkai T, Somech R, Broides A, et al. Late diagnosis of chronic granulomatous disease. Clin Exp Immunol 2020;201:297-305.

5. Kuhns DB, Alvord WG, Heller T, et al. Residual NADPH oxidase and survival in chronic granulomatous disease. $\mathrm{N}$ Engl J Med 2010;363:2600-10.

6. Winkelstein JA, Marino MC, Johnston RB Jr, et al. Chronic granulomatous disease. Report on a national registry of 368 patients. Medicine (Baltimore) 2000;79:155-69.

7. Liese JG, Jendrossek V, Jansson A, et al. Chronic granulomatous disease in adults. Lancet 1996;347:220-3.

8. Rawat A, Vignesh P, Sudhakar M, et al. Clinical, Immunological, and Molecular Profile of Chronic Granulomatous Disease: A Multi-Centric Study of 236 Patients From India. Front Immunol 2021;12:625320.

9. Akar HT, Esenboga S, Cagdas D, et al. Clinical and Immunological Characteristics of 63 Patients with Chronic Granulomatous Disease: Hacettepe Experience. J Clin Immunol 2021;41:992-1003.

10. Marciano BE, Rosenzweig SD, Kleiner DE, et al. Gastrointestinal involvement in chronic granulomatous disease. Pediatrics 2004;114:462-8.

11. Maddah M, Fazlollahi MR, Shiari R, et al. Lupus Erythematosus and Chronic Granulomatous Disease: Report of Four Iranian Patients with AR-CGD and One XL-CGD. Iran J Allergy Asthma Immunol 2019;18:452-8.
12. Keller MD, Notarangelo LD, Malech HL. Future of Care for Patients With Chronic Granulomatous Disease: Gene Therapy and Targeted Molecular Medicine. J Pediatric Infect Dis Soc 2018;7:S40-4.

13. Kohn DB, Booth C, Kang EM, et al. Lentiviral gene therapy for $\mathrm{X}$-linked chronic granulomatous disease. Nat Med 2020;26:200-6.

14. Bielorai B, Toren A, Wolach B, et al. Successful treatment of invasive aspergillosis in chronic granulomatous disease by granulocyte transfusions followed by peripheral blood stem cell transplantation. Bone Marrow Transplant 2000;26:1025-8.

15. Zhou S, Zhang R, Lv C, et al. Initial Dosage Optimization of Tacrolimus in Pediatric Patients With Thalassemia Major Undergoing Hematopoietic Stem Cell Transplantation Based on Population Pharmacokinetics. Ann Pharmacother 2021;55:440-51.

16. Gao Y, Ma J. Tacrolimus in adult hematopoietic stem cell transplantation. Expert Opin Drug Metab Toxicol 2019;15:803-11.

17. Ishiwata $Y$, Nagata $M$, Kiuchi $S$, et al. Intravenous Infusion of Fentanyl Has No Effect on Blood Concentration of Tacrolimus in Patients Receiving Hematopoietic StemCell Transplantation. Ther Drug Monit 2021;43:688-91.

18. Soskind R, Xiang E, Lewis T, et al. Initial tacrolimus weight-based dosing strategy in allogeneic hematopoietic stem-cell transplantation. J Oncol Pharm Pract 2021;27:1447-53.

19. Aftandilian C, Weinberg K, Willert J, et al. Invasive Fungal Disease in Pediatric Patients Undergoing Allogeneic Hematopoietic Stem Cell Transplant. J Pediatr Hematol Oncol 2016;38:574-80.

20. Yáñez L, Insunza A, Ibarrondo P, et al. Experience with anidulafungin in patients with allogeneic hematopoietic stem cell transplantation and graft-versus-host disease. Transpl Infect Dis 2015;17:761-7.

21. Iwamoto T, Monma F, Fujieda A, et al. Effect of Genetic Polymorphism of CYP3A5 and CYP2C19 and Concomitant Use of Voriconazole on Blood Tacrolimus Concentration in Patients Receiving Hematopoietic Stem Cell Transplantation. Ther Drug Monit 2015;37:581-8.

22. Imamura CK, Furihata $\mathrm{K}$, Okamoto $\mathrm{S}$, et al. Impact of cytochrome $\mathrm{P} 4502 \mathrm{C} 19$ polymorphisms on the pharmacokinetics of tacrolimus when coadministered with voriconazole. J Clin Pharmacol 2016;56:408-13.

23. Wang D, Chen X, Xu H, et al. Population pharmacokinetics and dosing regimen optimization of tacrolimus in Chinese pediatric hematopoietic stem cell 
transplantation patients. Xenobiotica 2020;50:178-85.

24. Yang JW, Liao SS, Zhu LQ, et al. Population pharmacokinetic analysis of tacrolimus early after Chinese pediatric liver transplantation. Int J Clin Pharmacol Ther 2015;53:75-83.

25. Wang DD, Chen X, Fu M, et al. Model extrapolation to a real-world dataset: evaluation of tacrolimus population pharmacokinetics and drug interaction in pediatric liver transplantation patients. Xenobiotica 2020;50:371-9.

26. Anderson BJ, Holford NH. Mechanism-based concepts of size and maturity in pharmacokinetics. Annu Rev Pharmacol Toxicol 2008;48:303-32.

27. Dedieu C, Albert MH, Mahlaoui N, et al. Outcome of chronic granulomatous disease - Conventional treatment vs stem cell transplantation. Pediatr Allergy Immunol 2021;32:576-85.

28. Magnani A, Mahlaoui N. Managing Inflammatory Manifestations in Patients with Chronic Granulomatous Disease. Paediatr Drugs 2016;18:335-45.

29. Martire B, Rondelli R, Soresina A, et al. Clinical features, long-term follow-up and outcome of a large cohort of patients with Chronic Granulomatous Disease: an Italian multicenter study. Clin Immunol 2008;126:155-64.

30. Cole T, Pearce MS, Cant AJ, et al. Clinical outcome in children with chronic granulomatous disease managed conservatively or with hematopoietic stem cell transplantation. J Allergy Clin Immunol 2013;132:1150-5.

31. Horwitz ME, Barrett AJ, Brown MR, et al. Treatment of chronic granulomatous disease with nonmyeloablative conditioning and a T-cell-depleted hematopoietic allograft. N Engl J Med 2001;344:881-8.

32. Seger RA, Gungor T, Belohradsky BH, et al. Treatment of chronic granulomatous disease with myeloablative conditioning and an unmodified hemopoietic allograft: a survey of the European experience, 1985-2000. Blood 2002;100:4344-50.

33. Salvator H, Mahlaoui N, Catherinot E, et al. Pulmonary manifestations in adult patients with chronic granulomatous disease. Eur Respir J 2015;45:1613-23.

34. Soncini E, Slatter MA, Jones LB, et al. Unrelated donor and HLA-identical sibling haematopoietic stem cell transplantation cure chronic granulomatous disease with good long-term outcome and growth. Br J Haematol 2009;145:73-83.

35. Seger RA. Hematopoietic stem cell transplantation for chronic granulomatous disease. Immunol Allergy Clin North Am 2010;30:195-208.

36. Cole T, McKendrick F, Titman P, et al. Health related quality of life and emotional health in children with chronic granulomatous disease: a comparison of those managed conservatively with those that have undergone haematopoietic stem cell transplant. J Clin Immunol 2013;33:8-13.

37. Arnold DE, Seif AE, Jyonouchi S, et al. Allogeneic hematopoietic stem cell transplantation in adolescent patients with chronic granulomatous disease. J Allergy Clin Immunol Pract 2019;7:1052-1054.e2.

38. Tang X, Zhang Y, Jing Y, et al. Allogeneic hematopoietic stem cell transplantation using unrelated cord blood or unmanipulated haploidentical donors is effective in pediatric chronic granulomatous disease with inflammatory complications and severe infection. Bone Marrow Transplant 2020;55:1875-8.

39. Rocha YC, López JÁ, Orrego JC, et al. Successful immune reconstitution by means of hematopoietic stem cell transplantation in a Colombian patient with chronic granulomatous disease. Biomedica 2016;36:204-12.

40. Morillo-Gutierrez B, Beier R, Rao K, et al. Treosulfanbased conditioning for allogeneic HSCT in children with chronic granulomatous disease: a multicenter experience. Blood 2016;128:440-8.

41. Segal BH, Veys P, Malech H, et al. Chronic granulomatous disease: lessons from a rare disorder. Biol Blood Marrow Transplant 2011;17:S123-31.

42. Wang DD, Chen X, Li ZP. Wuzhi capsule and haemoglobin influence tacrolimus elimination in paediatric kidney transplantation patients in a population pharmacokinetics analysis: A retrospective study. J Clin Pharm Ther 2019;44:611-7.

43. Chen X, Wang DD, Xu H, et al. Population pharmacokinetics and pharmacogenomics of tacrolimus in Chinese children receiving a liver transplant: initial dose recommendation. Transl Pediatr 2020;9:576-86.

44. Yonwises W, Wacharachaisurapol N, Anugulruengkitt $\mathrm{S}$, et al. Population pharmacokinetics of meropenem in critically ill infant patients. Int J Infect Dis 2021. [Epub ahead of print]. doi: 10.1016/j.ijid.2021.08.031.

45. Nassar-Sheikh Rashid A, Schonenberg-Meinema D, Berends SE, et al. Population pharmacokinetics of infliximab in children with juvenile idiopathic arthritis. Ther Drug Monit 2021. [Epub ahead of print]. doi: 10.1097/FTD.0000000000000914.

46. Hao GX, Huang X, Zhang DF, et al. Population pharmacokinetics of tacrolimus in children with nephrotic syndrome. Br J Clin Pharmacol 2018;84:1748-56.

47. Zhao W, Elie V, Baudouin V, et al. Population 
pharmacokinetics and Bayesian estimator of mycophenolic acid in children with idiopathic nephrotic syndrome. Br J Clin Pharmacol 2010;69:358-66.

48. Chen X, Wang DD, Xu H, et al. Initial dosage optimization of tacrolimus in Chinese pediatric patients undergoing kidney transplantation based on population pharmacokinetics and pharmacogenetics. Expert Rev Clin Pharmacol 2020;13:553-61.

(English Language Editor: A. Kassem)

Cite this article as: Chen $X$, Wang D, Lan J, Wang G, Zhu L, Xu X, Zhai X, Xu H, Li Z. Effects of voriconazole on population pharmacokinetics and optimization of the initial dose of tacrolimus in children with chronic granulomatous disease undergoing hematopoietic stem cell transplantation. Ann Transl Med 2021;9(18):1477. doi: 10.21037/atm-21-4124 